\title{
La Historia desde la perspectiva de los alumnos del Bachillerato Diversificado
}

Cristina Benítez, Daniel Gonda, Claudia Pereira

\section{DEFINICIÓN DEL PROBLEMA Y OBJETIVOS DE LA INVESTIGACIÓN}

En las últimas décadas del siglo XX estamos asistiendo a una serie de acontecimientos de todo orden como por ejemplo los referidos al vertiginoso avance científico y tecnológico. Por otra parte tenemos los que apuntan a la problemática humana, cada vez más compleja e inquietante que se vive a nivel mundial. ¿Porqué tanto avance científico y tecnológ ico no logra resolver la problemática humana y mejorar las condiciones de vida de la población?. ¿Qué características deberá tener esa educación, para que sea de calidad y responda a los desafíos que se le plantean en el aquí y ahora, y no quede relegada en la dinámica del mundo actual.?

Nos adherimos a lo planteado por Camino Trapero en el párrafo que citamos a continuación:

«...De acuerdo a la reflexión que venimos planteando, consideramos que la educación del futuro, no debe ser otra, que una educación de cuño humanista, centrada en la persona, más allá de la eficiencia 0 de la eficacia, sin que esto se descarte. El eje será ayudar a la persona a crecer en todas las dimensiones de su ser: individual, social y trascendente. En suma, una educación que posibilite al ser humano el desarrollo del pensamiento crítico, la libertad responsable, la conciencia axiológica y el compromiso con la comunidad». ${ }^{1}$

(Camino Trapero, 1993. pág 21).

Nuestra actividad pedagógica, nos ha permitido a lo largo de los años, detectar con preocupación, la escasa importancia que los estudiantes de enseñanza secundaria adjudican al conocimiento de carácter humanístico a portado por las materias tales como Historia, Literatura, Filosofía y Sociología. La rapidez de los cambios en la sociedad, tanto en el aspecto cognitivo como en el tecnológico, tiende a formar generaciones más tecnócratas, en las cuales la importancia del conocimiento aportado por las materias Historia, Filosofía y Literatura es sensiblemente desjerarquizado por parte de los estudiantes de enseñanza secundaria, independientemente de su orientación.

Nuestra investigación se centra en la importancia que adjudican los estudiantes de cuarto y quinto año del Bachillerato Diversificado de Enseñanza Secundaria, al conocimiento humanístico aportado por el estudio de la materia curricular Historia.

\section{PROPUESTA DE TRABAJO}

¿Ven los estudiantes siempre una aplicación inmediata del conocimiento que reciben a través de la materia curricular Historia, independientemente de la orientación que escojan?

¿Consideran los estudiantes al conocimiento aportado por la materia Historia de relevancia para resolver problemas de la vida cotidiana?

\section{Objetivo General}

Investigar la importancia que los alumnos le dan al conocimiento humanístico aportado por Historia como materia curricular en los cuartos y quintos años del Bachillerato Diversificado (segundo ciclo de Enseñanza Secundaria).

\section{Objetivo Específico}

Tratar de indagar si en las concepciones de los estudiantes del segundo ciclo de enseñanza media se percibe una aplicación inmediata del conocimiento humanístico aportado por el estudio de la materia curricular Historia. 


\section{Metodolocía}

La presente investigación se enmarca dentro de las estrategias cualitativas de generación de conocimiento. Nuestro objetivo fue tratar de entender cual es el universo de concepciones de los alumnos de cuarto y quinto año de Enseñanza Secundaria, en relación al conocimiento humanístico aportado por la materia curricular Historia.

Dada las características de la misma, se decidió utilizar un método de investigación cualitativo, y se consideró que el método fenomenográfico, era el que mejor se adaptaba a los objetivos propuestos. La fenomenografía es un método de investigación cualitativo, descriptivo y a diferencia del método positivista es inductivo.

Al respecto podemos citar a Thomas Kroksmark (1987, p227):

«La fenomenografía es inductiva, lo que significa que la investigación trata particularidades específicas cuya expresión es procesada y elevada a conclusiones generales sobre el objeto estudiado y examinado. Lo contrario es el método deductivo, donde el investigador formula una teoría sobre un fenómeno para posteriormente probar la validez de la misma en las particularidades específicas»².

La fenomenografía realiza una distinción entre el mundo objetivo, con todos sus elementos visibles y observables, y el mundo experimentado subjetivamente.

Este método de investigación, parte de la base de que existe un universo limitado de concepciones sobre los distintos fenómenos, (Alberto Nagle. 1988) ${ }^{3}$. Se entiende por concepción, la idea básica sobre un fenómeno, constituye el conjunto de ideas básicas que subyacen detrás de nuestras expresiones y formas de relacionarnos con un fenómeno. La concepción, reside pues, en el territorio de lo que se da por sobreentendido.

El método fenomenográfico es un intento cualitativo, de comprender y revelar la perspectiva del individuo, es decir, revelar la perspectiva de segundo orden.

La perspectiva de segundo orden es la que constituye el objeto de análisis; es la concepción, antes que el mundo objetivo con todos sus elementos observables lo que focalizan los fenomenógrafos en sus trabajos. (Nagle 1988 op.cit).

Otro rasgo característico del método fenomenográfico lo constituye el hecho de que el resultado final de la empresa de investigación, está dado por la obtención de un sistema de categorías. El sistema de categorías representa un universo de concepciones cualitativamente diferentes sobre un mismo fenómeno. Este universo de concepciones es limitado. Las categorías en la medida que representan distintas formas de pensar, pueden ser utilizadas en otro contexto diferente al que le dio nacimiento.

«...Cada categoría constituye una parte potencial de una estructura mayor y a su vez relacionada a otras categorías de descrip ción. La fenomenografía tiene como meta, descubrir el sistema estructural dentro de que coexisten diferentes categorías interpretativas...»

«...Un sistema de categorías, a pesar de haber sido originadas contextualmente son descontextualizadas y pueden resultar de utilidad en contextos diferentes al que las ha generado. La fenomenografía tiene como meta descubrir el sistema estructural dentro del que coexisten diferentes categ orías interpretativas. Dichas estructuras podrían resultar de utilidad para entender como entienden las personas...»4. (Marton Ference, 1986. pág. 34).

Para nuestra investigación utilizamos como instrumento de colecta de datos, la entrevista semiestructurada. Por medio de ella pretendimos aprehender la perspectiva del sujeto entrevistado sobre el tema investigado.

Los sujetos de investigación fueron alumnos del primer y segundo año del Bachillerato Diversificado, pertenecientes a un liceo público y otro privado. El total de los sujetos de investigación fue de treinta y cuatro alumnos, cuyas edades oscilan entre 15 y 17 años, y se distribuyeron de la siguiente forma: doce alumnos pertenecientes a cuarto año de un liceo privado, doce alumnos pertenecientes a cuarto año de un liceo público y diez alumnos pertenecientes a quinto año de un liceo público. 


\section{PRESENTACIÓN DE RESULtADOS}

Del procesamiento de las distintas colectas de datos realizadas en los diferentes liceos surgió un sistema de categorías que representa formas cualitativamente diferentes de entender o concebir la asignatura Historia en el contexto de la Educación Secundaria del país. A continuación se presenta el sistema de categorías.

\section{A. El Concepto De Temporalidad En La Historia}

Determinados alumnos afirman que la Historia, es una herramienta para entender el presente a la luz del pasado. En ésta categoría, los alumnos, analizan los sucesos del presente, relacionando su origen con hechos del pasado. Los alumnos analizan determinados acontecimientos históricos y los conectan con la problemática del mundo contemporáneo. El estudio del programa de cuarto año ( primer año del bachillerato diversificado), les llama la atención, ya que se relaciona con la Historia contemporánea, pudiéndose explicar diferentes situaciones de injusticia social, conflictos bélicos, reclamos territoriales, así como el eterno conflic to entre el Capitalismo y el Socialismo.

\section{B. Problematizar la Asignatura, Es Inducir A Pensarla.}

Estos estudiantes valoran y aprecian las clases interactivas, alejándose del modelo tradicional de clase frontal. Una vez más, se puede comprobar que el estudiante, en la medida en que se ve estimulado a participar en el trabajo, en la clase, adquiere mayor interés por lo que en ella se está desarrollando. Una clase que sé desarrolla en un ambiente de respeto y calidez, favorece el intercambio entre el docente facilitador y los alumnos. Los estudiantes están más dispuestos para el razonamiento, buscando así la causalidad entre los diferentes sucesos que se han dado en el tiempo.

Otro aspecto que se puede destacar es la importancia que tiene la personalidad y el carisma del docente, en el momento en que al alumno debe valorar la importancia del conocimiento humanístico, aportado por la materia curricular Historia.

\section{Capacidad De Integrar los Temas De Historia, Dentro Del Curriculum.}

Los estudiantes que incluimos en ésta categoría son capaces de visualizar que determinados temas pueden ser enfocados desde diferentes perspectivas, eliminando las barreras de la compartimentación curricular.

Esta particularidad, (la de poder considerar un mismo tema desde diferentes ópticas), no es frecuente que se pueda describir en estudiantes pertenecientes al primer año del Bachillerato Diversificado (común a todas las orientaciones), ya que el tipo de educación que se venía impartiendo en el país, hasta hace unos años, en cierta forma inducía a la creencia de que un mismo tema, sólo podía ser considerado desde una sola perspectiva.

Actualmente se está creando un nuevo sistema de estudio, que lleva al alumno a considerar un mismo tema, bajo diferentes ópticas. Esta forma de encarar los temas, trata de formar mentes más inquisid oras, con mayor capacidad de asombro, y por lo tanto mayor tendencia a la investigación, a la búsqueda de los elementos comunes o dispares.

\section{La Historia Como Generadora De Cultura.}

Estudiantes que ven a la historia como una materia importante para su formación cultural. En esta categoría surgieron distintas características, que nos describen como nuestros estudiantes perciben el conocimiento aportado por la materia curricular Historia. Aparece aquí, la Historia como cultura general, ayudando así al estudiante a integrarse a la sociedad en que está inmerso; junto con este concepto, encontramos el concepto de cotidianeidad, hecho al cual todo ser humano está ligado, sea cual fuere la actividad que le toque desempeñar en la división social del trabajo. 


\section{E. La Historia Analiza Al Mundo Desde Distintas Perspectivas.}

Los estudiantes reconocen la importancia de la historia como asignatura para su formación. Ellos sin embargo enfatizan, con diferentes grados de rigidez, distintos aspectos de la misma. Por ejemplo el acento en la historia nacional o universal. Las preguntas centrales son las siguientes: ¿Cómo visitar ese extraño país llamado pasado ? ¿Cómo entender lo sucedido ?¿Cómo se enriquece el estudiante a través del conocimiento de distintos aspectos de la realidad histórica?

\section{F. La Historia Como Generadora De Conocimientos Para Entender la ReALidad.}

La historia como un medio para entender la realidad. El común denominador de esta concepción es el aporte de la historia a los individuos para ambientarse en el mundo que lo rodea. En esta categoría, los estudiantes son conscientes de la importancia del conocimiento de los hechos sociales, políticos y económicos más trascendentes para entender la realidad en la que están inmersos

\section{CONCLUSIONES}

El análisis empírico nos ha permitido extraer las siguientes conclusiones:

La mayoría de los educandos percibe el estudio de la asignatura Historia, como la disciplina que fundamentalmente brinda las herramientas necesarias que permiten interpretar y comprender hechos y circunstancias del presente.

Se trata de un conocimiento humanístico de relevancia, toda vez que propende a la integración social de los individuos. Es interesante hacer notar, que aparece un concepto recurrente en todas las entrevistas: la Historia es vista como una herramienta de interpretación de hechos pasados, que ayudan a entender la problemática actual.

No faltó la concepción de quienes piensan (tal vez por deficiencias en el método de enseñanza, o de divulgación de la asignatura) que la asignatura no facilita la resolución de problemas que presenta la realidad actual. Estos alumnos ven a la Historia , como una mera interpretación de los hechos del pasado, aislados de la realidad actual.

Consideramos que debe ser el presente, el punto de partida para el estudio de la materia, para así facilitar la comprensión de la sociedad en la que los alumnos están inmersos. De esta manera los alumnos podrán tener una visión más clara del por qué de la historia.

Somos conscientes, de que la Historia no puede ser totalmente objetiva, ya que el historiador está viviendo un tiempo y lugar quizás distinto al momento que le toca recrear. Es inevitable por lo tanto, que dicha narración no tenga un tinte personal colmado de las vivencias de su narrador y por supuesto de su audiencia.

De cualquier manera creemos en las palabras de Claxton cuando dice:

«La cuestión es que, sea lo que sea que los estudiantes se lleven consigo, deberá ser verdaderamente útil por derecho propio» (Claxton, 1989).

Este grupo modestamente intentó rescatar las ideas de nuestros estudiantes sobre el conocimiento humanístico, y así aportar un granito de arena para que lo que nuestros estudiantes se lleven consigo, les sea realmente útil para su vida futura.

${ }^{1}$ Trapero, C. (1993) «Educación en valores». Montevideo, Barreiro y Ramos.

${ }^{2}$ Kroksmark, T. (1987) «Fenómeno Grafisk Didaktik». Góteb orgs, Acta Universitatis Gothoburgensis. ${ }^{3}$ Nagle, A. (1988) «Orientación Vocacional: una investigación desde los jóvenes uruguayos». Gotemburgo, Góteborgs Universitatis.

${ }^{4}$ Marton, F. (1986) «Phenomenography a research approach to investigating different und erstanding of reality» en J ournal of Thought, 21. 